\title{
Serovars distribution and antimicrobial resistance patterns of Salmonella spp. isolated from the swine farms and slaughter houses
}

\author{
Hokyoung Jung, Sungseok Lee, Chiyoung Kim, Sunyoung Sunwoo, Young S. Lyoo* \\ College of Veterinary Medicine, Konkuk University, Seoul 143-701, Korea \\ (Accepted: April 14, 2011)
}

\begin{abstract}
Salmonella spp. is an important pathogen to both public and swine industry. The aim of this study was to investigate the distribution of Salmonella serovar and antibiotics susceptibility of the isolates from Korean swine producing systems. A total of 63 (5.28\%) Salmonella spp. was isolated from 1,194 samples ( 977 fecal materials and 67 organ samples). The predominant Salmonella (S.) enterica serotype and serovar was group B (69.8\%) and $S$. Typhimurium (47.6\%), $S$. Derby (20.6\%) and $S$. Heidelberg (1.6\%). But $S$. Cholerasuis which is characterized host specific by septicemia and enteritis to pigs was not isolated. Antimicrobial susceptibility of the isolates varies as follows: Norfloxacine (75\%), Ciprofloxacin (67.5\%), Amikacin (60\%), Colistin (60\%), Enrofloxacin (55\%). All of isolates were resistant to Erythromycin, Penicillin, Tetracycline and Lincomycin. The results of this study provided useful information regarding antimicrobial susceptibility and resistance patterns to treat salmonellosis and to prevent emergence of multidrug resistance Salmonella.
\end{abstract}

Keywords : antimicrobial, multidrug, resistance, Salmonella, serovar

\section{Introduction}

Salmonella spp. is an important pathogen to both public and swine industry [20]. Salmonellosis in swine and poultry is considered as a source of human infection because carcasses can be contaminated through contact with feces, blood, intestine contents of infected animals [4]. The genus of Salmonella is a gram negative bacillus and divided over 2,500 different serotypes. Some Salmonella serovars can affect multiple host species and it makes a serious problem according to the food chain [21, 22]. Salmonella spp. was isolated in the farms, slaughterhouse and food markets and the isolates' serovars were variable.

Recent report revealed a prevalence of Salmonella from slaughter house consisting, $13.5 \%$ of 2,732 lymph nodes and $4.4 \%$ of 1,118 cecal contents, respectively [15]. In another report, Salmonella was isolated from $15.72 \%$ to $21.25 \%$ of pigs in the all groups over the 30 days of age in Korean swine farms, and serovars of $22 \%$ were Salmonella (S.) Typhimurium or $S$. Cholerasuis [14].

Antibiotic administration for prevention and treatment in the infected animals are widely practicing in swine industry. But unfortunately, antibiotic resistances were resulted from the overuse of antibiotics, which is pertaining to serious concern on food safety of the animal product [3]. Currently, many advanced countries which implement good welfare and public health policy, prohibit some antibiotics as growth promoters in animal feedstuff $[5,8]$. Because, the frequent use of antibiotics increases the risk of the emergence of multi-drug resistant bacteria from food producing animals and possibly increases chances of these mutants transferring to humans [27]. A number of authors reported that Salmonella spp. with multi- or single-antimicrobial resistances was frequently isolated in pork production system [17-19].

The purpose of this study was to investigate the distribution of Salmonella serovar and antibiotics susceptibility of the isolates from Korean swine producing systems.

\section{Materials and Methods}

\section{Study design and collection of samples}

In order to investigate the distribution of Salmonella

\footnotetext{
*Corresponding author

Tel: +82-2-450-3719, Fax: +82-2-450-3037

E-mail: lyoo@konkuk.ac.kr
} 
serovar, the study was performed using serological and bacteriological methods in the swine farms and slaughter houses. The 62 farms and 2 slaughter houses were randomly selected from a list of swine farms according to their geographical location and herd size. At the selected farms four fecal samples to cross sectional study were collected from each of the compartment which were divided into five groups according to the age of pigs; $4,8,12,18,25$ weeks ( \pm 1 week) and sows. Approximately $10 \mathrm{~g}$ of fresh feces which were selected randomly on the floor of each pens were collected into the sterilized tube.

Organ samples including liver, gall bladder, and cecum were obtained during autopsies of pigs showing clinical signs in the farms and from post-evisceration of carcasses in the slaughter houses. Then, all samples were transported to the laboratory for the isolation of Salmonella spp. by microbiological culture methods [25].

\section{Bacteriological culture and serotyping}

For fecal samples, a sample was inoculated and cultured in 1\% buffered peptone water (BPW, 1:10 w/ v) at $37^{\circ} \mathrm{C}$ for $16 \mathrm{~h}$. Afterwards, $100 \mu \mathrm{L}$ of culture fluid were transferred into the Rappaport and Vassiliadis broth (RV, 1:100 v/v, MERK) and incubated at $42^{\circ} \mathrm{C}$ for 24 $\mathrm{h}$, and this procedure was repeated for all fecal samples. Colonies from selective enrichment medium were streaked on Salmonella shigella (SS) agar, xylose lysine deoxycholate (XLD) agar, Rambach agar and MacConkey agar plates, and incubated at $37^{\circ} \mathrm{C}$ for $18 \mathrm{~h}$. The presence of Salmonella isolates were confirmed by biochemical tests using BBL crystal ID system (BD, USA) and serological tests. The organ samples except gall bladder sac were homogenized and then inoculated by the same procedure described above.

All isolates were serotyped by agglutination test according to the Kauffmann-White scheme using Salmonella polyvalent somatic (O) and flagella $(\mathrm{H})$ antiserum (DIFCO, USA) [9].

\section{Antimicrobial susceptibility}

Antibiotics susceptibility of the Salmonella isolates was tested by the agar diffusion method, using 20 kinds of Sensidisk (BBL, USA) according to the method described by National Committee for Clinical Laboratory Standards.

Antibiotics disks used for the test were: amikacin $(\mathrm{An})$, ampicillin (Am), amoxicillin-clavulanic acid (Amc), carbenicillin $(\mathrm{Cb})$, cefazolin $(\mathrm{Cz})$, cephalothin $(\mathrm{Cf})$, ciprofloxacin (Cip), chloramphenicol (C), colistin (Cl), erythromycin (E), enrofloxacin (Enr), gentamicin (Gm), kanamycin (K), lincomycin (L), neomycin $(\mathrm{N})$, norfloxacin (Nor), oxytetracycline (T), penicillin (P), streptomycin (S) and trimethoprime (Tmp). The reference bacteria such as Staphylococcus aureus (ATCC 25923) and Escherichia coli (ATCC 25922) were used for quality control of the test.

\section{Salmonella Cholerasuis - specific polymerase chain reaction (PCR)}

Salmonella Cholerasuis genes were amplified by PCR following the protocol of a previous study [6]. Briefly, $1 \mathrm{~mL}$ of the final bacteriological culture broths was centrifuged to harvest enriched bacteria at $8,000 \mathrm{rpm}$ for $5 \mathrm{~min}$. The harvested cells were lysed by boiling at $100^{\circ} \mathrm{C}$ for $10 \mathrm{~min}$ and then centrifugated at $4,000 \mathrm{rpm}$ for $30 \mathrm{sec}$.

Five microliters of the supernatant were mixed with the PCR premix (iNtRON Biotechnology, Korea; included i-Taq DNA polymerase $2.5 \mathrm{U}$, dNTPs $2.5 \mathrm{mM}$ ), each 10 pmol of sense and antisense primers, and added diethyl pyrocarbonate (DEPC) treated water to $20 \mu \mathrm{L}$. $S$. cholerasuis ATCC 13312 and distilled water were used as positive and negative control. PCR conditions were initial denaturation at $94^{\circ} \mathrm{C}$ for $10 \mathrm{~min}$, and then 35 cycle of $94^{\circ} \mathrm{C}$ for $30 \mathrm{sec}, 55^{\circ} \mathrm{C}$ for $30 \mathrm{sec}$, and $72^{\circ} \mathrm{C}$ for $1 \mathrm{~min}$, and final extension at $72^{\circ} \mathrm{C}$ for $7 \mathrm{~min}$. Expect size of amplicons is $963 \mathrm{bp}$ in length.

\section{Results}

A total of 977 fecal materials and 67 organ samples was collected and analyzed for Salmonella infection in pigs. Salmonella spp. was recovered from 63 of 1,194 samples (5.28\%). More specifically, Salmonella spp. was isolated from $1.5 \%$ of fecal sample from the cross section study, $19.4 \%$ of organ samples and $19.3 \%$ of fecal samples at holding area in the slaughter house (Table 1).

The predominant Salmonella enterica serotype was group B (69.8\%) which comprised of $S$. Typhimurium (47.6\%), S. Derby (20.6\%) and $S$. Heidelberg (1.6\%), followed by group C $S$. Rissen (17.5\%) and group E1 $S$. London. The most frequent isolates serovar was $S$. Typhimurium, but $S$. Cholerasuis (group C) was neither isolated in the bacterial culture nor detected by PCR (Table 2). 
Table 1. Isolation frequency of Salmonella spp.

\begin{tabular}{lcccc}
\hline \hline & Cross section study & Organ sample & Slaughter house & Total \\
\hline No. of samples (No. of farms) & $941(48)$ & $103(22)$ & $150(15)$ & $1,194(85)$ \\
No. of isolated salmonella (No. of Farm) & $14(6)$ & $20(5)$ & $29(10)$ & $63(21)$ \\
Recovery rate \% (Farm level \%) & $1.5(12.5)$ & $19.4(22.7)$ & $19.3(66.7)$ & $5.28(24.7)$ \\
\hline
\end{tabular}

Table 2. Distribution of Salmonella $(S$.) serovar by serum agglutination test

\begin{tabular}{llrc}
\hline \hline \multicolumn{2}{l}{ O group } & No. of bacteria & Isolate rate (\%) \\
\hline \multirow{3}{*}{ B } & $S$. Typhimurium & 30 & 47.6 \\
& $S$. Derby & 13 & 20.6 \\
& $S$. Heidelberg & 1 & 1.6 \\
\hline C & $S$. Rissen & 11 & 17.5 \\
\hline E1 & $S$. London & 1 & 1.6 \\
\hline \multirow{2}{*}{ Other } & $-^{*}$ & 7 & 11.1 \\
\hline
\end{tabular}

${ }^{*}$ The isolates which un-defined serovar are followed as serogroup B (5 isolates), D1 (1 isolate) and unknown serogroup (1 isolate).
As for the results of antibiotics susceptibility test 75\% of the isolates were susceptible to Nor. Antimicrobial susceptibility of the isolates varies as follows; Cip (67.5\%), An (60\%), Cl (60\%), Enr (55\%), G (55\%), respectively. The isolates were highly susceptible to Quinolone antibiotics such as Cip and Nor, except Enr. On the other hand the isolated Salmonella strains showed resistance to a few antibiotics such as E, P, T, L (Table 3).

The result of antimicrobial resistance pattern analysis in 40 isolates revealed various and different resistance combinations of 3 to 15 antimicrobials. The three frequent antimicrobial resistance patterns were CfELTP (22.5\%), AmAmcCbCfCEGmKLNTPSTmp (7.5\%) and AmAmc CbCzCfCipCEEnrLTPSTmp (7.5\%), respectively. The

Table 3. Antimicrobial susceptibility of Salmonella spp. isolated from pigs

\begin{tabular}{|c|c|c|c|c|c|}
\hline \multirow{2}{*}{ Antibiotics } & \multirow{2}{*}{$\mathrm{n}$} & \multicolumn{3}{|c|}{ Prevalence } & \multirow{2}{*}{-Susceptibility (\%) } \\
\hline & & Resistance & Intermediate & Susceptibility & \\
\hline Amikacin (An) & 40 & 5 & 11 & 24 & 60.0 \\
\hline Ampicillin (Am) & 40 & 22 & 4 & 14 & 35.0 \\
\hline Amoxicillin-clavulanic acid (Amc) & 40 & 22 & 3 & 15 & 37.5 \\
\hline carbenicillin $(\mathrm{Cb})$ & 40 & 26 & 14 & 0 & 0.0 \\
\hline cefazolin $(\mathrm{Cz})$ & 40 & 17 & 7 & 16 & 40.0 \\
\hline Cephalothin (Cf) & 40 & 36 & 3 & 1 & 2.5 \\
\hline Ciprofloxacin (Cip) & 40 & 4 & 9 & 27 & 67.5 \\
\hline Chloramphenicol (C) & 40 & 16 & 5 & 19 & 47.5 \\
\hline Colistin $(\mathrm{Cl})$ & 40 & 1 & 15 & 24 & 60.0 \\
\hline Erythromycin $(\mathrm{E})$ & 40 & 40 & 0 & 0 & 0.0 \\
\hline Enrofloxacin (Enr) & 40 & 14 & 4 & 22 & 55.0 \\
\hline Gentamicin $(\mathrm{Gm})$ & 40 & 16 & 2 & 22 & 55.0 \\
\hline Kanamycin (K) & 40 & 11 & 16 & 13 & 32.5 \\
\hline Lincomycin $(\mathrm{L})$ & 40 & 40 & 0 & 0 & 0.0 \\
\hline Neomycin $(\mathrm{N})$ & 40 & 11 & 23 & 6 & 15.0 \\
\hline Norfloxacin (Nor) & 40 & 0 & 10 & 30 & 75.0 \\
\hline Oxytetracycline $(\mathrm{T})$ & 40 & 39 & 0 & 1 & 2.5 \\
\hline Penicillin $(\mathrm{P})$ & 40 & 40 & - & 0 & 0.0 \\
\hline Streptomycin (S) & 40 & 28 & 9 & 3 & 7.5 \\
\hline Trimethoprime (Tmp) & 40 & 19 & 5 & 16 & 40.0 \\
\hline
\end{tabular}

An: $30 \mu \mathrm{g}, \mathrm{An}: 10 \mu \mathrm{g}$, Amc: $30 \mu \mathrm{g}, \mathrm{Cb}: 100 \mu \mathrm{g}$, Cz: $30 \mu \mathrm{g}$, Cf: $30 \mu \mathrm{g}$, Cip: $5 \mu \mathrm{g}, \mathrm{C}: 30 \mu \mathrm{g}, \mathrm{Cl}: 10 \mu \mathrm{g}, \mathrm{E}: 15 \mu \mathrm{g}, \mathrm{Enr}: 5 \mu \mathrm{g}$, Gm: $10 \mu \mathrm{g}, \mathrm{K}: 30 \mu \mathrm{g}, \mathrm{L}: 2 \mu \mathrm{g}, \mathrm{N}: 30 \mu \mathrm{g}$, Nor: $10 \mu \mathrm{g}$, T: $30 \mu \mathrm{g}, \mathrm{P}: 10 \mu \mathrm{g}, \mathrm{S}: 10 \mu \mathrm{g}$, Tmp: $5 \mu \mathrm{g}$. 
Table 4. Antimicrobial resistance patterns of the Salmonella spp.

\begin{tabular}{|c|c|c|}
\hline No. of antibiotics (resistance \%) & Resistance pattern & Number of isolate \\
\hline $3(2.5 \%)$ & ELP & 1 \\
\hline $4(2.5 \%)$ & ELTP & 1 \\
\hline $5(22.5 \%)$ & CfELTP & 9 \\
\hline $6(2.5 \%)$ & EGmLTPS & 1 \\
\hline \multirow{2}{*}{$7(5.0 \%)$} & CfCELTPS & 1 \\
\hline & CbCfEKLPS & 1 \\
\hline $8(2.5 \%)$ & CbCELTPSTmp & 1 \\
\hline \multirow{2}{*}{$9(5.0 \%)$} & AmAmcCbCfELTPTmp & 1 \\
\hline & AnCfClEL NTPS & 1 \\
\hline \multirow{2}{*}{$10(5.0 \%)$} & AmAmcCbCfELTPSTmp & 1 \\
\hline & CbCfCipCEEnrLTPS & 1 \\
\hline $11(5.0 \%)$ & AmAmcCbCzCf EGmLTPS & 2 \\
\hline $12(2.5 \%)$ & AnAmAmcCbCfELNTPSTmp & 1 \\
\hline \multirow{2}{*}{$13(10.0 \%)$} & $\underline{\mathrm{AmAmcCbCzCfCEEnrGmLTPS}}$ & 2 \\
\hline & $\overline{\mathrm{AmAmcCbCzCf}}$ CEEnrLTPSTmp & 2 \\
\hline \multirow{3}{*}{$14(17.5 \%)$} & $\overline{\text { AmAmcCbCf CEGmKLNTPSTmp }}$ & 3 \\
\hline & AmAmcCbCzCfCipCEEnrLTPSTmp & 3 \\
\hline & $\overline{\mathrm{AnAmAmcCbCz}} \mathrm{Cf}$ EEnrGmLT PSTmp & 1 \\
\hline \multirow{6}{*}{$15(17.5 \%)$} & AmAmcCbCzCfCEEnrGmKLTPSTmp & 2 \\
\hline & AmAmcCbCzCf$C E G m K L N T \underline{P S T m p}$ & 1 \\
\hline & AmAmcCbCzCf EEnrGmKLNT PSTmp & 1 \\
\hline & AmCbCzCfCEEnrGmKLNTPSTmp & 1 \\
\hline & An $\underline{A m A m c C b C z C f C E E n r G m K L T P S}$ & 1 \\
\hline & AnAmcCbCzCf CEGmKLNTPSTmp & 1 \\
\hline Total (100\%) & & 40 \\
\hline
\end{tabular}

The underline indicate $\mathrm{AmAmcCbCzCfP}$ resistance pattern. The shadow indicate CfELTP resistance pattern.

isolates which contain beta-lactam antimicrobials Resistance pattern (AmAmcCbCzCfP) was found in 15 isolates $(37.5 \%)$ (Table 4).

\section{Discussion}

In this study, Salmonella was isolated from fresh feces, cecal contents and organs including lung, liver, gall bladder and cecum. The average of recovery rate was $5.28 \%(63 / 1,194)$ of total samples which consist of $1.5 \%(14 / 941)$ of fecal samples from the cross section study in the farm level, 19.4\% (20/103) of organ samples and $19.3 \%(29 / 150)$ of fecal samples in slaughter houses. The prevalence of Salmonella spp. at farm level was $12.5 \%(6 / 48)$ for cross section study, $22.7 \%$ (5/22) for organ samples and $66.7 \%$ (10/15) for pigs at slaughter houses. Prevalence of Salmonella from organs (autopsied in the farm) and at slaughter houses was higher than that in the cross section study. The differences in recovery rates in the present study indicate that the shedding of
Salmonella augments by the poor health condition of pigs; Salmonella infected pigs could act as subclinical carrier when influenced by stress factors such as noise, mixing with other groups, high stock density, change of environment, which subsequently increase the number of excreted Salmonella spp. present in the feces [26].

The serovars of 56 isolates were confirmed as $S$. Typhimurium, $S$. Derby, $S$. Rissen, $S$. Heidelberg and $S$. London by standard typing method. And the other 7 isolates were defined partially that just defined the type of somatic antigens $(\mathrm{O})$. Among the isolates, the serogroup $\mathrm{B}$ was the most frequent $(69.8 \%)$ and followed by the serogroup C (17.5\%, S. Rissen). There are two similar papers about the sero-prevalence of Salmonella in Korea. Lee et al. [15] and Kim et al. [14] have reported that the most frequently isolated salmonella was serogroup B which showed $69.5 \%$ and $69.3 \%$, respectively. Some Salmonella serovars are cause a serious disease depending to host animal. The results in present study showed higher prevalence rates than those in these previous 
reports. S. enterica serovar group $\mathrm{B}$ and $\mathrm{C} 1$ are commonly isolated in swine farm and slaughter house. Group D1 is frequently isolated in poultry industry.

In this study, the most commonly isolated serotype was $S$. Typhimurium (33.3\%). This serovar is very important to the public health, because it is a zoonotic bacterium and frequently isolated from the swine production system. Similar results were reported in the prevalence surveys for Salmonella at the swine farm or slaughter house in Japan [10, 13], Italy [16], Spain [11], Ireland [17, 23], Denmark [2] and the USA [12]. These similar results which show that $S$. Typhimurium is widely distributed over the world emphasize, the needs for a standard management protocol to avoid introduction and transmission of Salmonella in swine herds and slaughter houses. When the salmonella infected pigs are transported into slaughter house, they could excrete the pathogen at the lairage, contaminate carcasses during the slaughtering process and residues with spray dust in the space of slaughterhouses [1, 28]. $S$. Cholerasuis is condemned as the serovar which was commonly isolated in the swine farms [7]. But in this study, S. Cholerasuis was not determined in isolation by culture and detection of specific gene (flic) by PCR (the data was not shown).

The present study demonstrated that the antimicrobial resistance and emergence of multidrug resistance were seriously higher than in the past years or in other countries [12, 14, 15]. Lee et al. [15] reported that 315 Salmonella isolates from Dec. 2000 to Nov. 2001 in Korean slaughter houses were not resistant $(<1 \%)$ to $\mathrm{Gm}$, Amc, Cz, Cf, Cl, Cip and Nor. In contrast, Kim et al. [14] reported that 114 Salmonella spp. isolated in 2005 in Korean swine farms were resistant to $\mathrm{T}(100 \%)$, P (100\%), Am (92.98\%), Sulfametoxazol/Tmp (89.47\%) and L/S (81.58\%). Rayamajhi et al. [24] showed the resistance to $\mathrm{S}(94.1 \%)$, T $(90.1 \%)$, Am (64.7\%), C (56.8\%) and $\mathrm{Gm}(54.9 \%)$. In comparison to the finding of the previous studies showed that Salmonella's resistance tends to increase and become more complex.

The antimicrobial resistance patterns in the present study could be classified to the 24 patterns which consist of antimicrobials combinations from 3 to 15 different kinds of drugs. The major antimicrobial resistance pattern was CfELTP (22.5\%). The CfELTP including resistance pattern was $85 \%$ in 40 isolates. The other pattern combination of frequent resistant antimicrobial was the pattern including AmAmcCbCfELTPSTmp (37.5\%). In the similar study, Huang et al. [12] showed that the resistance pattern in 197 Salmonella isolates from 2003 to $2005,27.4 \%$ of isolates were not resistant or resistant to one antimicrobial (Florfenicol, Spectinomycin, Tetracycline) among 13 antimicrobials. GarcíaFeliz et al. [11] reported that less than $10 \%$ of the isolates were resistant to Amc, N, Cf, Apramycin and $\mathrm{Gm}$, and multi-resistance(resistant to four or more drugs) was detected from more than $50 \%$ of the isolates in Spain from Mar. 2003 to Feb. 2004. But in this study, the minimum resistance pattern was three antimicrobials resistance (ELP).

\section{Conclusion}

The results of this study provided useful information regarding antimicrobial susceptibility and resistance patterns to treat salmonellosis and to prevent emergence of multidrug resistance bacteria.

\section{References}

1. Alban L, Stärk KD. Where should the effort be put to reduce the Salmonella prevalence in the slaughtered swine carcass effectively? Prev Vet Med 2005, 68, 6379.

2. Baggesen DL, Wegener HC, Bager F, Stege H, Christensen J. Herd prevalence of Salmonella enterica infections in Danish slaughter pigs determined by microbiological testing. Prev Vet Med 1996, 26, 201213.

3. Berends BR, van den Bogaard AE, Van Knapen F, Snijders JM. Human health hazards associated with the administration of antimicrobials to slaughter animals. Part II. An assessment of the risks of resistant bacteria in pigs and pork. Vet Q 2001, 23,10-21.

4. Botteldoorn N, Herman L, Rijpens N, Heyndrickx M. Phenotypic and molecular typing of Salmonella strains reveals different contamination sources in two commercial pig slaughterhouses. Appl Environ Microbiol 2004, 70, 5305-5314.

5. Castanon JIR. History of the use of antibiotic as growth promoters in European poultry feeds. Poult Sci 2007, 86, 2466-2471.

6. Chiu TH, Pang JC, Hwang WZ, Tsen HY. Development of PCR primers for the detection of Salmonella enterica serovar Choleraesuis based on the fliC gene. J Food Prot 2005, 68, 1575-1580. 
7. Clothier KA, Kinyon JM, Frana TS. Comparison of Salmonella serovar isolation and antimicrobial resistance patterns from porcine samples between 2003 and 2008 . J Vet Diagn Invest 2010, 22, 578-582.

8. Dibner JJ, Richards JD. Antibiotic growth promoters in agriculture: history and mode of action. Poult Sci 2005, 84, 634-643.

9. Difco Laboratory. Serological identification of Salmonella. Difco Laboratory, Michigan, 1977.

10. Futagawa-Saito K, Hiratsuka S, Kamibeppu M, Hirosawa T, Oyabu K, Fukuyasu T. Salmonella in healthy pigs: prevalence, serotype diversity and antimicrobial resistance observed during 1998-1999 and 2004-2005 in Japan. Epidemiol Infect 2008, 136, 1118-1123.

11. García-Feliz C, Collazos JA, Carvajal A, Herrera S, Echeita MA, Rubio P. Antimicrobial resistance of Salmonella enterica isolates from apparently healthy and clinically ill finishing pigs in Spain. Zoonoses Public Health 2008, 55, 195-205.

12. Huang TM, Lin TL, Wu CC. Serovar distribution and antimicrobial susceptibility of swine Salmonella isolates from clinically ill pigs in diagnostic submissions from Indiana in the United States. Lett Appl Microbiol 2009, 48, 331-336.

13. Kawagoe K, Mine H, Asai T, Kojima A, Ishihara K, Harada K, Ozawa M, Izumiya H, Terajima J, Watanabe H, Honda E, Takahashi T, Sameshima T. Changes of multi-drug resistance pattern in Salmonella enterica subspecies enterica serovar typhimurium isolates from food-producing animals in Japan. $\mathrm{J}$ Vet Med Sci 2007, 69, 1211-1213.

14. Kim EM, Kim HK, Park SJ, Lee CS, Luo Y, Moon HJ, Yang JS, Park BK. Prevalence and antimicrobial resistance patterns of Salmonella spp. Isolated from different aged pigs in Korea. Korean J Vet Res 2007, 47, 395-398

15. Lee WW, Jung BY, Kim HT, Chung KT, Lee GR, Kim KH, Lee DS, Kim YH. Prevalence and antimicrobial susceptibility of Salmonella isolated from Korean slaughter pigs. Korean J Vet Serv 2003, 26, 313-321

16. Lomonaco S, Decastelli L, Bianchi DM, Nucera D, Grassi MA, Sperone V, Civera T. Detection of Salmonella in finishing pigs on farm and at slaughter in Piedmont, Italy. Zoonoses Public Health 2009, 56, 137-144.
17. McDowell SW, Porter R, Madden R, Cooper B, Neill SD. Salmonella in slaughter pigs in Northern Ireland: prevalence and use of statistical modelling to investigate sample and abattoir effects. Int $\mathrm{J}$ Food Microbiol 2007, 118, 116-125.

18. Mejía W, Casal J, Zapata D, Sánchez GJ, Martín M, Mateu E. Epidemiology of salmonella infections in pig units and antimicrobial susceptibility profiles of the strains of Salmonella species isolated. Vet Rec 2006, 159, 271-276.

19. Molla B, Berhanu A, Muckle A, Cole L, Wilkie E, Kleer J, Hildebrandt G. Multidrug resistance and distribution of Salmonella serovars in slaughtered pigs. J Vet Med B Infect Dis Vet Public Health 2006, 53, 28-33.

20. Murray CJ. Salmonellae in the environment. Rev Sci Tech 1991, 10, 765-785.

21. Pfeifer CG, Marcus SL, Steele-Mortimer O, Knodler LA, Finlay BB. Salmonella typhimurium virulence genes are induced upon bacterial invasion into phagocytic and nonphagocytic cells. Infect Immun 1999, 67, 5690-5698.

22. Popoff MY, Bockemühl J, Gheesling LL. Supplement 2001 (no. 45) to the Kauffmann-White scheme. Res Microbiol 2003, 154,173-174.

23. Prendergast DM, Duggan SJ, Gonzales-Barron U, Fanning S, Butler F, Cormican M, Duffy G. Prevalence, numbers and characteristics of Salmonella spp. on Irish retail pork. Int J Food Microbiol 2009, 131, 233-239.

24. Rayamajhi N, Kang SG, Kang ML, Lee HS, Park KY, Yoo HS. Assessment of antibiotic resistance phenotype and integrons in Salmonella enterica serovar Typhimurium isolated from swine. J Vet Med Sci 2008, 70, 1133-1137.

25. Ruiz J, Sempere MA, Varela MC, Gomez J. Modification of the methodology of stool culture for Salmonella detection. J Clin Microbiol 1992, 30, 525-526.

26. Schwartz KJ. Salmonellosis. In: Taylor DJ, Straw BE, D'Allaire S, Mengeling WL, (eds.). Disease of Swine. 8th ed. pp. 535-551, Blackwell, Oxford, 1999.

27. Tollefson L, Flynn WT. Impact of antimicrobial resistance on regulatory policies in veterinary medicine: status report. AAPS PharmSci 2002, 4, E37.

28. Williams LP, Newell KW. Patterns of Salmonella excretion in market swine. Am J Public Health Nations Health 1967, 57, 466-471. 